\section{a) gag_1}

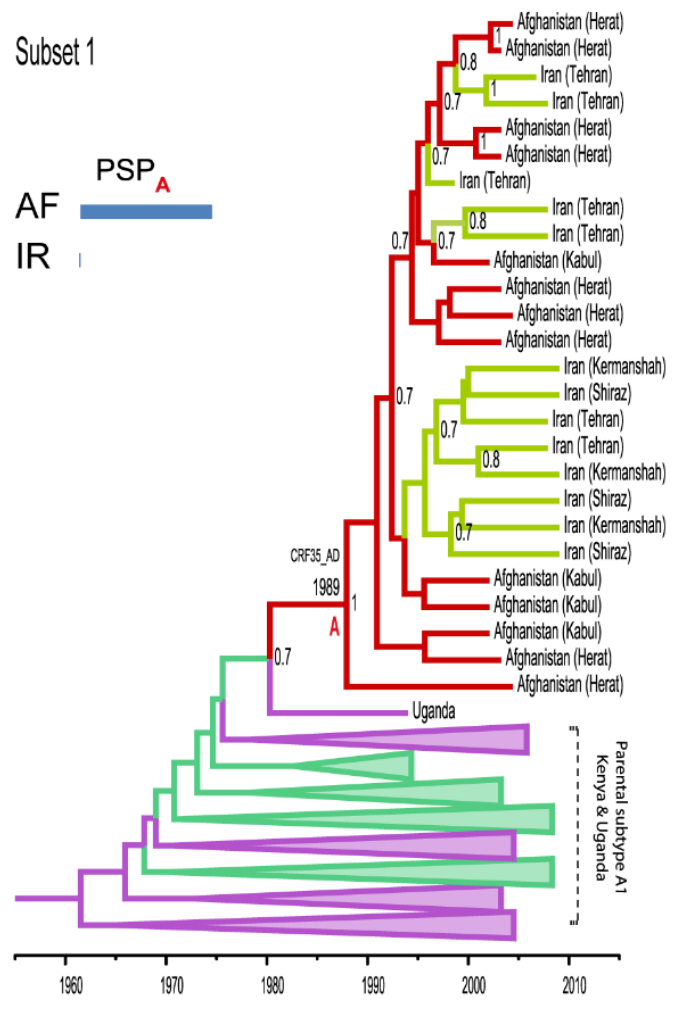

Subset3

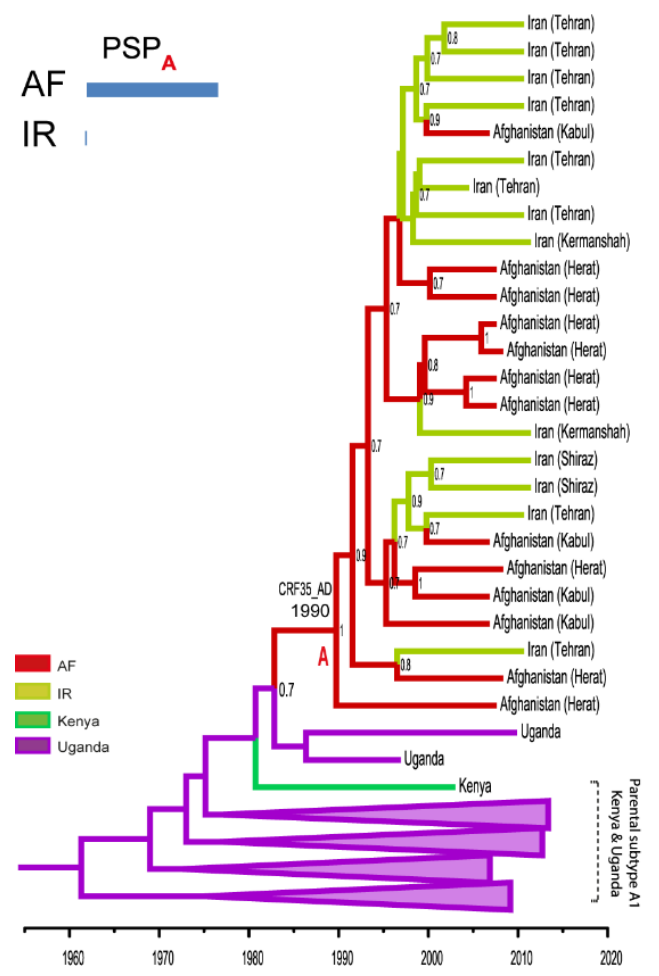

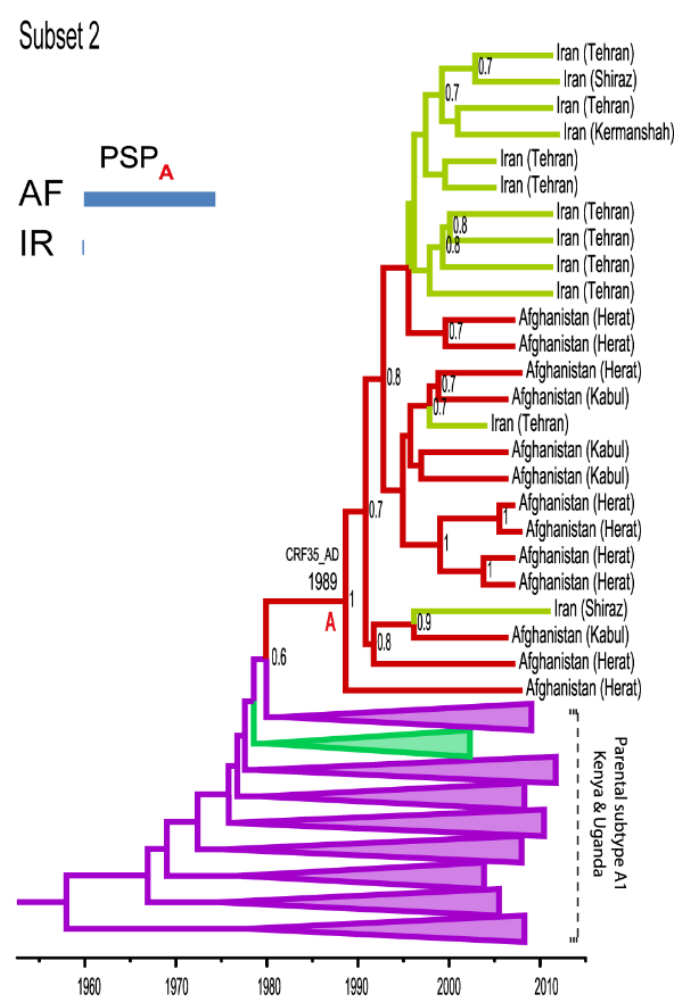

Subset 4

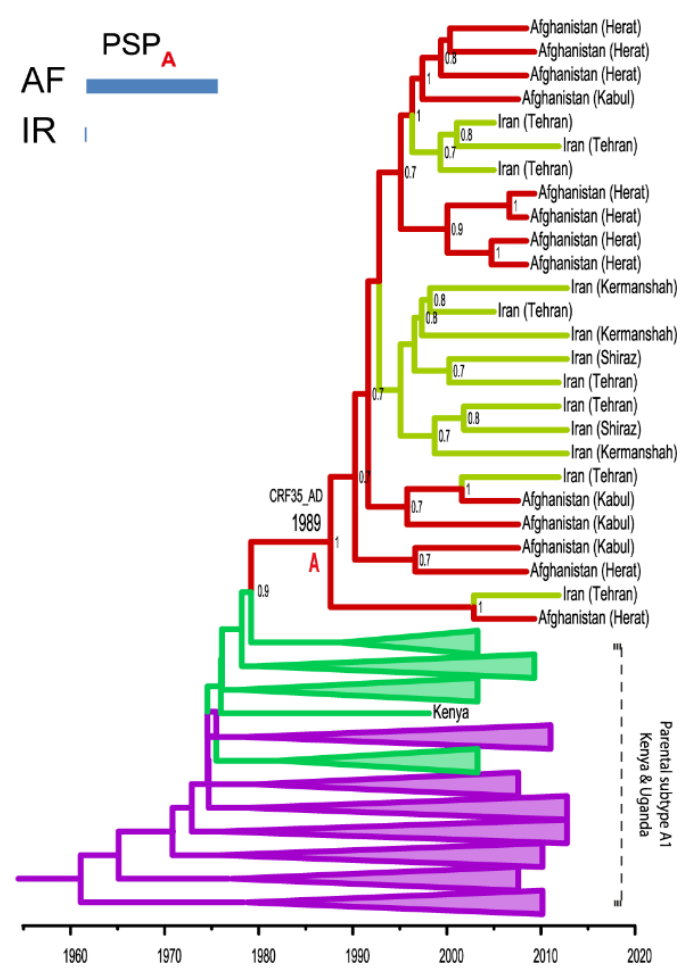




\section{b) gag_2}
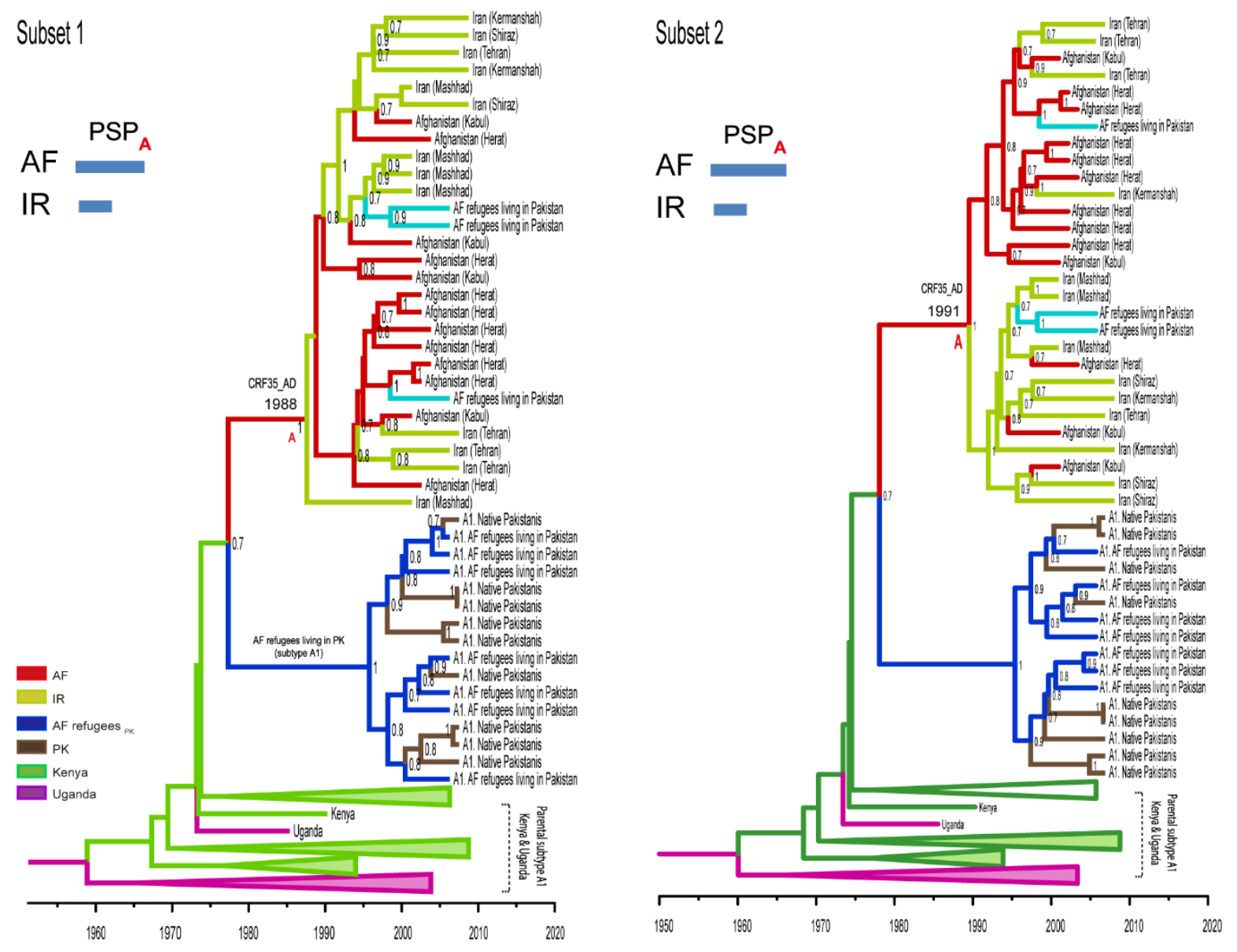

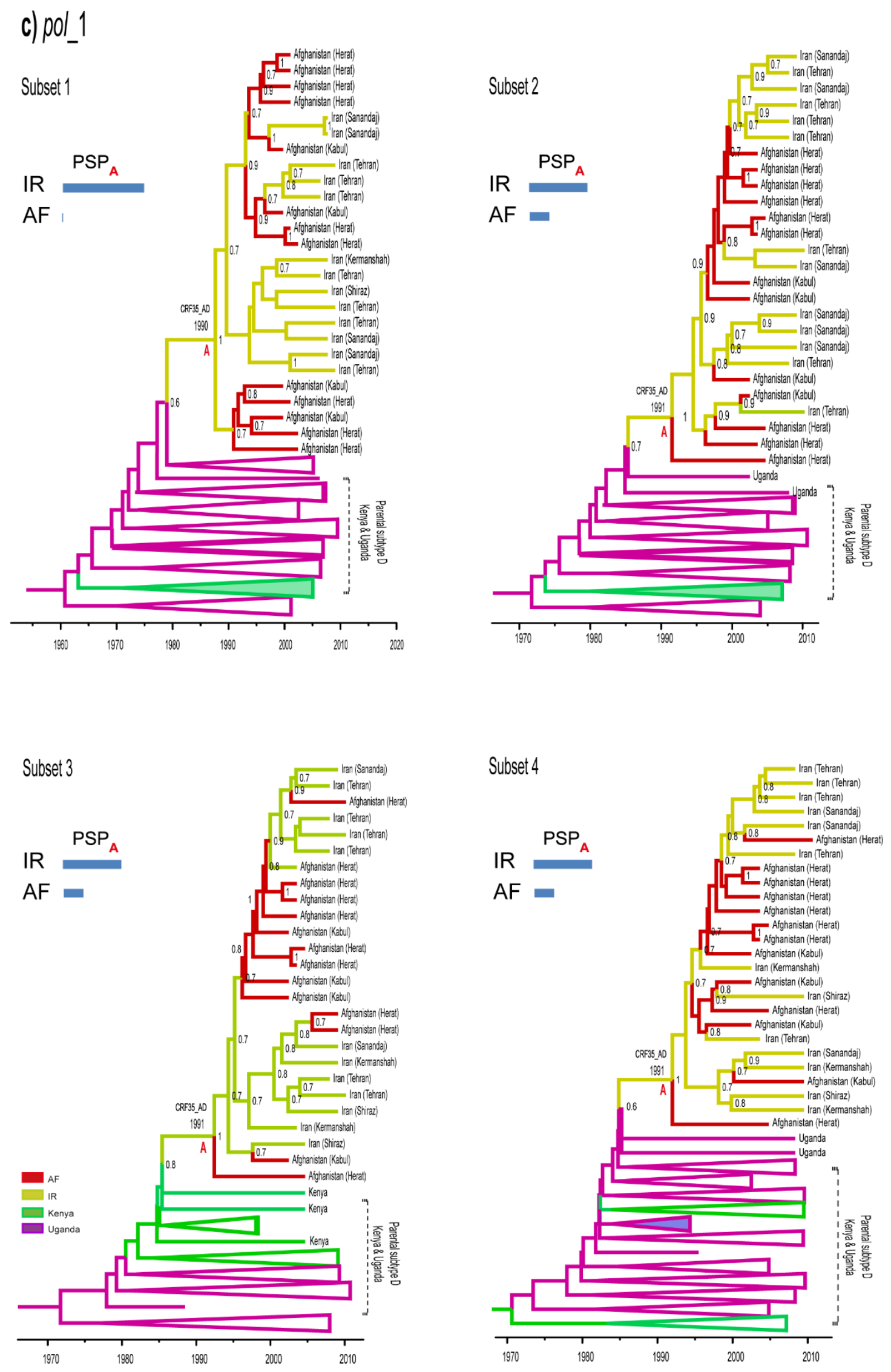

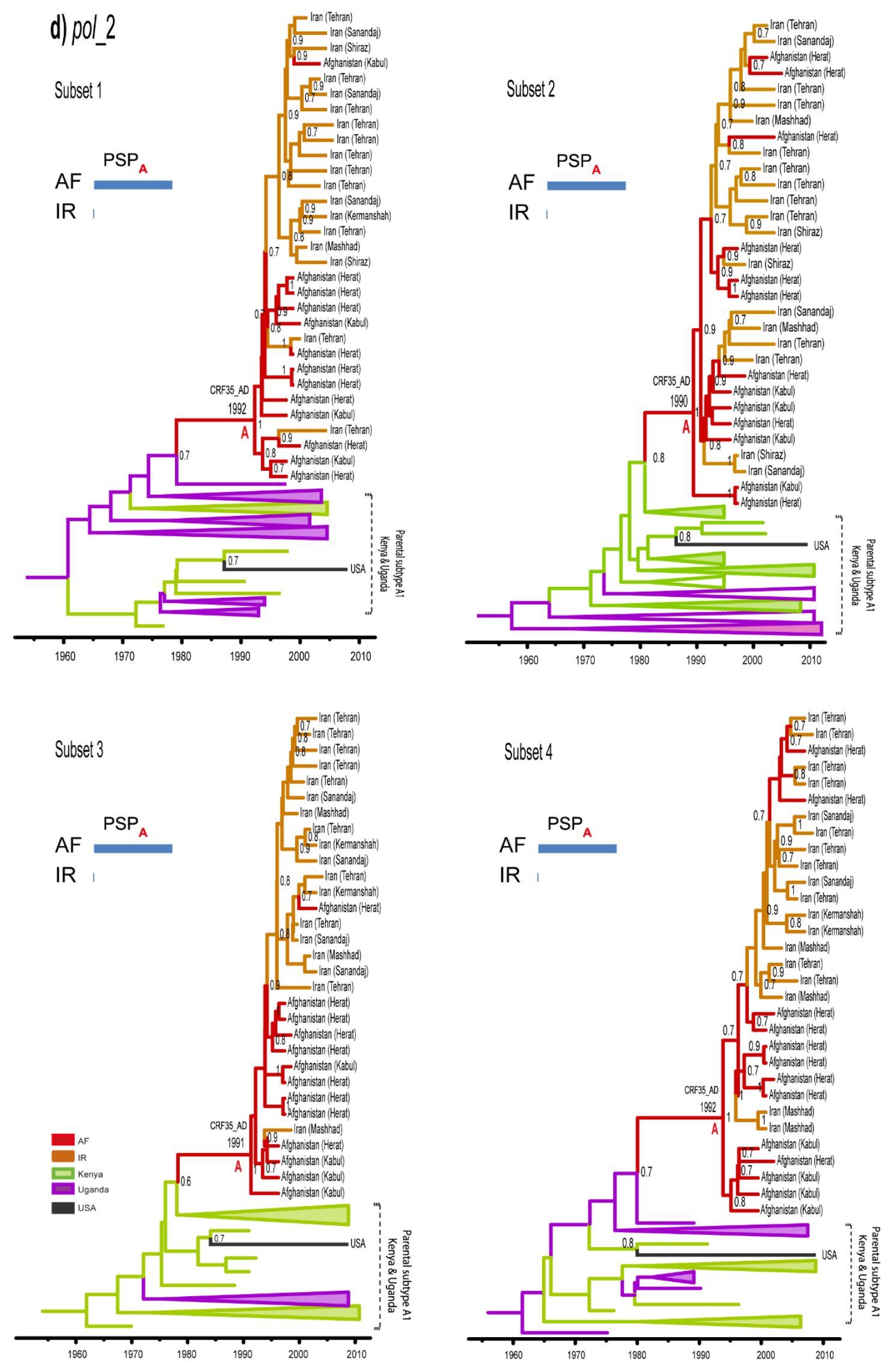
S7 Fig. Robustness of the key parameters' estimates to the choice of different datasets. (a) gag_1; (b) gag_2; (c) pol_1; (d) pol_2. As shown in the figure, the key parameters are robust to the choice of different datasets, including the tree topology and height, the $T_{\text {mrca }}$ of the CRF35_AD Afghan-Iranian $_{\text {cluster }}$ (see also Fig S5), dispersion pattern of the virus between Afghanistan and Iran, the evolutionary history of the North American CRF35_AD-like strain, and the linkage between CRF35_AD strains identified among Afghan refugees and the CRF35_AD epidemic in Afghanistan and Iran. Similar to the complete analyses, we could not clearly identify the country (i.e., Afghanistan or Iran) that first established or received the CRF35_AD epidemic. In the gag_2 region, the total number of sequences available from Iran was small $(n=21)$; therefore, only two balanced subsets were created and analyzed for this region. For each genomic region, the color code is indicated in the legend, on the lower left corner. To the upper left of each phylogeny, probable ancestral locations with their posterior probabilities are indicated for the CRF35_A Afghan-Iranian cluster (Node A). Posterior clade credibility values greater than 0.7 are indicated for the key nodes. Parental sequences are collapsed for visual clarity. PSP: Posterior State Probability; A1: HIV-1 subtype A1; D: HIV-1 subtype D; AF: Afghanistan; IR: Iran; AF refugees рк: Afghan refugees living in Pakistan 